\title{
Assessing the Impact of Isoniazid Preventive Therapy (IPT) on Tuberculosis Incidence and Predictors of Tuberculosis among Adult Patients Enrolled on ART in Nekemte Town, Western Ethiopia: A Retrospective Cohort Study
}

\author{
Gemechu Tiruneh $\mathbb{D}^{1},{ }^{1}$ Alemayehu Getahun, ${ }^{2}$ and Emiru Adeba ${ }^{2}$ \\ ${ }^{1}$ Department of Medical Laboratory Sciences, College of Medical and Health Sciences, Wollega University, Nekemte, \\ P.O. Box: 395, Ethiopia \\ ${ }^{2}$ Department of Public Health, College of Medical and Health Sciences, Wollega University, Nekemte, P.O. Box: 395, Ethiopia \\ Correspondence should be addressed to Gemechu Tiruneh; gemechu333@gmail.com
}

Received 26 October 2018; Accepted 10 February 2019; Published 2 May 2019

Academic Editor: Subhada Prasad Pani

Copyright (C) 2019 Gemechu Tiruneh et al. This is an open access article distributed under the Creative Commons Attribution License, which permits unrestricted use, distribution, and reproduction in any medium, provided the original work is properly cited.

\begin{abstract}
Background. Isoniazid preventive therapy is a prophylactic treatment used in the prevention of active tuberculosis. It is known to be most effective in preventing tuberculosis in patients with positive tuberculin skin test. Methods. A retrospective cohort study centering on two institutions in Nekemte town, Western Ethiopia, was employed. Secondary data of 600 medical records were analyzed by Cox regression. Result. Tuberculosis incidence among the Isoniazid treated group was 1.98 per 100 person-years and 4.52 per 100 person-years in the untreated group. CD4 cell count, clinical staging, body mass index (BMI), not using cotrimoxazole, body weight, and functional status were significant predictors of tuberculosis risk. Isoniazid preventive therapy use was associated with 55\% reduction of tuberculosis incidence. Conclusion. Isoniazid preventive therapy use was associated with significant reduction in tuberculosis incidence, even in the absence of Tuberculin Skin Test (TST). Therefore, isoniazid preventive therapy (IPT) coverage should be used more widely, with special emphasis given to patients at higher risk of tuberculosis. The study shows that the absence of TST testing should not be a limitation.
\end{abstract}

\section{Background}

Tuberculosis $(\mathrm{TB})$ is the commonest of all opportunistic infections in people living with Human Immune Deficiency Virus (HIV) and causes preventable Acquired Immune Deficiency Syndromes (AIDS) related mortality and morbidity, especially in sub-Saharan Africa. The risk of acquiring TB for HIV positive individuals is $26-28$ times greater than in HIV negative individuals [1]. The high rate of TB in HIV infected individuals gives rise to the need for strategies to prevent $\mathrm{TB}$ in this population. The World Health Organization (WHO) recommends isoniazid preventive therapy (IPT) as part of the three I's strategies for TB/HIV coinfection: IPT, intensified TB case finding, and infection control for TB $[1,2]$. IPT is a proven strategy for reducing $\mathrm{TB}$ in individuals at a community and population level. The effectiveness of IPT in preventing TB has been well established in HIV-negative individuals and communities as well as in HIV-infected populations [2-4]. The greatest benefit of IPT in preventing TB is clearly established for people with confirmed latent TB infection which is diagnosed by a positive TST $[5,6]$.

In Ethiopia, TST is not applicable to initiate IPT. Therefore, initiation of IPT is based on symptomatic screening to rule out the presence of active TB; this could limit the effectiveness of IPT by missing those with latent TB[68]. This study aims to review the effectiveness of IPT in a country like Ethiopia where TST and other techniques used to diagnose latent tuberculosis infections (LTBI) are absent. Adherence to IPT is important and poor adherence will limit its impact. Unlike other studies which include 
nonadherent patients in their assessment of IPT impact, this study only compares patients who complete a full course of treatment with an untreated group. Furthermore, this study will identify other factors that favor the occurrence of TB which may be useful in identifying areas of future focus in the control and management of TB among patients living with HIV.

\section{Methods}

2.1. Study Setting and Context. The study was a facility-based retrospective cohort study, with a three-year followup, using clinical data from adult HIV patients receiving ART/HAART (antiretroviral therapy/highly active antiretroviral therapy) at two health facilities in Nekemte town: Nekemte referral hospital and Nekemte health center. The health facilities are pioneers in providing chronic HIV care services in Ethiopia. A total of 7500 adult HIV patients receive ART across both institutions, of which 5000 are based at Nekemte referral hospital [9].

2.2. Study Population. The study was based on secondary data retrieved from clinical registers of patients starting HIV care between 2009 and 2012. The subjects included in the study were selected based on eligibility criteria set for IPT exposed and unexposed cohorts. The cohorts were defined based on whether the patient had received IPT, which was regarded as the primary exposure variable of the cohorts. Patients who had completed six months of isoniazid prophylaxis were classified in the exposed cohort while patients who had never been offered IPT were considered unexposed. Adult patients enrolled on ART, aged 18 and above, who were free of active TB and who had completed six months of Isoniazid therapy were considered eligible candidates and were included in the exposed group. For the unexposed cohort, patients who had never initiated IPT and who met all other previously stated criteria were included.

2.3. Sample Size and Sampling Procedure. The required sample size was determined using Epi-info Version 7 assuming an independent cohort study. It was assumed that Isoniazid completers were exposed throughout the followup and untreated groups were not exposed. The incidence of TB amongst IPT treated groups was $4.36 \%$ and $12 \%$ for non-IPT groups, taken from a former study [8]. Power of $80 \%$ and $5 \%$ margin of error, with exposed to unexposed ratio of 2:1, was taken into account. Therefore, the final sample size was 400 for unexposed and 200 for the exposed group. Qualified candidates were sorted based on predefined inclusion criteria for both groups. IPT registration logbook was used as a frame to screen the exposed group and the ART registration logbook for the unexposed group. The study subjects were selected for each group independently using a simple random sampling technique where purposive serial numbers were assigned to each of the presorted patient folders. The final study population was selected using a lottery method until the predetermined sample size was attained for each cohort.
2.4. Variables and Data Collection Procedures. The primary exposure variable was IPT receipt while incidence of TB was the main outcome variable. Sociodemographic and clinical variables were considered in order to identify predictors of TB incidence during the followup process. Those patients who were lost to followup, study cessation year, $10^{\text {th }}$ of September 2012, died, or who transferred out of area were considered as censorship states during the analysis. In the non-IPT group (unexposed) those who initiated IPT prophylaxis were censored on the date of IPT initiation. Every patient was followed until the occurrence of TB or any of the censorship states which occurred first.

2.5. Data Analysis. Data was entered, sorted, doublechecked, and analyzed using SPSS version 20. Univariate analysis was performed to show the summary of each variable of the entire cohort using a simple frequency table. To identify predictors of $\mathrm{TB}$ incidence, every variable in the bivariate analysis of Cox proportional hazard model with crude hazard ratio of $\mathrm{p}$-value $<0.2$ was selected to build the multivariate model. Each variable was checked for confounding effect against IPT and change in regression coefficient of each variable by less than $20 \%$ when compared with a crude model revealed absence of confounding. Interaction effect or effect modification was also assessed to check whether the effect of IPT was modified when variables were included in the multivariate model henceforth; all interaction models were statistically insignificant. Universal assumptions of Cox proportional hazard model was tested by graphical methods using log-log plot. The assumption of Cox proportional hazard model was not violated and the result is valid at specified significance level and degree of freedom.

\section{Results}

3.1. Baseline Sociodemographics and Clinical Description of the Cohorts. A total cohort of $600 \mathrm{HIV}$ patients on ART, comprising 200 IPT and 400 non-IPT, were followed for a median duration of 26 (IQR 20-33) months. 356 (59\%) of the patients were female. Mean baseline age of the both cohorts was 33 years old with SD 9. Half (51\%) were married at baseline, and $106(18.43 \%)$ widowed. The main religious group was Ethiopian Orthodox, comprising 237(41\%), and $154(27 \%)$ of the cohorts were educated up to secondary school level (Table 1).

The median CD4 count at baseline for the entire cohort was 381 (IQR 262-535). 517(86.16\%) of the cohort had adequate functional status at baseline to perform their daily duties. The majority $(76.33 \%)$ of the entire cohort were categorized as WHO clinical stage I or II at the start of followup. $60 \%$ of the study subjects had a $\mathrm{BMI} \geq 18.5$ at enrollment while $56.3 \%$ of the entire cohorts were higher than $50 \mathrm{~kg}$ at baseline (Table 2).

3.2. Incidence of TB. 600 study participants were followed for a total of 1482.55 person-years of observation. 53 (8.8\%) of participants developed TB while in followup and 547 individuals were censored from the study, giving an overall 
TABLE 1: Baseline sociodemographic characteristics of PLWH at Nekemte referral hospital and Nekemte Health center followed from September 2009-September 2012.

\begin{tabular}{|c|c|c|c|c|}
\hline \multirow{2}{*}{ Variables } & \multirow{2}{*}{ Category } & \multicolumn{2}{|c|}{ Cohort Group } & \multirow[t]{2}{*}{ Total N (\%) } \\
\hline & & $\begin{array}{c}\text { IPT Cohort } \\
\mathrm{n}(\%)\end{array}$ & $\begin{array}{c}\text { Non-IPT } \\
\mathrm{n}(\%)\end{array}$ & \\
\hline \multirow{4}{*}{ Age Group $(\mathrm{n}=600)$} & $18-30$ & $102(51 \%)$ & $199(49.75 \%)$ & $301(50.16 \%)$ \\
\hline & $31-40$ & $68(34 \%)$ & $140(35 \%)$ & $208(34.6)$ \\
\hline & $41-50$ & $25(12.5 \%)$ & $44(11 \%)$ & $69(11.5 \%)$ \\
\hline & $>50$ & $5(2.5 \%)$ & $17(4.25 \%)$ & $22(3.66 \%)$ \\
\hline \multirow{2}{*}{$\operatorname{Sex}(n=600)$} & Female & $113(56.5 \%)$ & $243(60.8 \%)$ & $356(59.6 \%)$ \\
\hline & Male & $87(43.5 \%)$ & $157(39.2 \%)$ & $244(40.7 \%)$ \\
\hline \multirow{4}{*}{ Religion $(\mathrm{n}=575)$} & Muslim & $37(18.8 \%)$ & $83(21.33 \%)$ & $120(20.5 \%)$ \\
\hline & Orthodox & $73(37.24 \%)$ & $164(42.16 \%)$ & $237(40.5 \%)$ \\
\hline & Protestant & $82(41.8 \%)$ & $130(33.42 \%)$ & $212(36.24 \%)$ \\
\hline & Others* & $4(2.04 \%)$ & $12(3.08 \%)$ & $16(2.73 \%)$ \\
\hline \multirow{4}{*}{ Educational Status $(\mathrm{n}=580)$} & No Education & $39(20.21 \%)$ & $89(22.94 \%)$ & $106(18.43)$ \\
\hline & Primary Education & $84(43.75 \%)$ & $160(41.28 \%)$ & $128(22.07 \%)$ \\
\hline & Secondary Education & $59(30.73 \%)$ & $95(24.48 \%)$ & $154(26.55 \%)$ \\
\hline & Tertiary Education & $10(5.2 \%)$ & $44(11.34 \%)$ & $54(9.3 \%)$ \\
\hline \multirow{4}{*}{ Marital Status $(\mathrm{n}=575)$} & Married & $93(48.19 \%)$ & $200(52.35 \%)$ & $293(50.96 \%)$ \\
\hline & Single & $39(20.21 \%)$ & $60(15.70 \%)$ & $99(17.28 \%)$ \\
\hline & Divorced/Separated & $26(13.48 \%)$ & $51(13.35 \%)$ & $77(13.39 \%)$ \\
\hline & Widowed/Widower & $35(18.13 \%)$ & $71(18.59 \%)$ & $106(18.43 \%)$ \\
\hline
\end{tabular}

* refers to Catholic or Adventist.

TABLE 2: Baseline clinical, laboratory results, and followup outcome characteristics of PLWH at Nekemte referral hospital and Nekemte Health center followed from September 2009-September 2012.

\begin{tabular}{|c|c|c|c|c|}
\hline \multirow{2}{*}{ Variables $(\mathrm{n}=600)$} & \multirow{2}{*}{ Category } & \multicolumn{2}{|c|}{ Cohort group } & \multirow[t]{2}{*}{ Total n (\%) } \\
\hline & & IPT n (\%) cohort & Non-IPT cohort n (\%) & \\
\hline \multirow{4}{*}{$\mathrm{CD} 4$} & $<=200$ & $20(10 \%)$ & $68(11.33 \%)$ & $88(14.66 \%)$ \\
\hline & $201-350$ & $64(32 \%)$ & $119(29.75 \%)$ & $183(30.5 \%)$ \\
\hline & $351-500$ & $52(26 \%)$ & $97(24.25 \%)$ & $149(24.83 \%)$ \\
\hline & $>500$ & $64(32 \%)$ & $116(29 \%)$ & $180(30 \%)$ \\
\hline \multirow{2}{*}{ BMI } & $>=18.5$ & $127(63.5 \%)$ & $229(57.25 \%)$ & $356(59.33 \%)$ \\
\hline & $<18.5$ & $73(36.5 \%)$ & $171(42.75 \%)$ & $244(40.66 \%)$ \\
\hline \multirow{2}{*}{ Weight } & $<=50$ & $85(42.5 \%)$ & $177(44.25 \%)$ & $266(40.66 \%)$ \\
\hline & $>50$ & $115(57.5 \%)$ & $223(55.75 \%)$ & $338(56.33 \%)$ \\
\hline \multirow{2}{*}{ WHO Stage } & $\mathrm{I} / \mathrm{II}$ & $164(82 \%)$ & $294(73.5 \%)$ & $458(76.33 \%)$ \\
\hline & III/IV & $36(18 \%)$ & $106(26.5 \%)$ & $142(23.66 \%)$ \\
\hline \multirow{2}{*}{ Functional Status } & Working & $169(84.5 \%)$ & $348(87 \%)$ & $517(86.16 \%)$ \\
\hline & Ambulatory/Bedridden & $29(14.5 \%)$ & $43(10.75 \%)$ & $72(18 \%)$ \\
\hline \multirow{2}{*}{ Opportunistic Infection } & Yes & $45(22.5 \%)$ & $80(20 \%)$ & $125(20.83 \%)$ \\
\hline & No & $155(77.5 \%)$ & $320(80 \%)$ & $475(79.16 \%)$ \\
\hline \multirow{2}{*}{ Cotrimoxazole Treatment } & Yes & $142(71 \%)$ & $287(71.75 \%)$ & $429(71.5 \%)$ \\
\hline & No & $58(29 \%)$ & $113(28.25 \%)$ & $171(28.5 \%)$ \\
\hline \multirow[t]{2}{*}{ Previous TB } & Yes & $45(22.5 \%)$ & $80(20 \%)$ & $125(20.83 \%)$ \\
\hline & No & $155(77.5 \%)$ & $320(80 \%)$ & $475(79.16 \%)$ \\
\hline \multirow{2}{*}{ Outcome status } & TB & $11(5.5 \%)$ & $42(10.5 \%)$ & $53(8.83 \%)$ \\
\hline & Censored & $189(94.5 \%)$ & $358(89.5 \%)$ & $547(91.16 \%)$ \\
\hline
\end{tabular}

Censored: loss to followup, death, transferred out, started IPT (for non-IPT). 
TABLE 3: Incidence rate of tuberculosis of PLWH at Nekemte referral hospital and Nekemte Health center followed from September 2009September 2012.

\begin{tabular}{|c|c|c|c|c|}
\hline Variables $(\mathrm{n}=600)$ & Category & Incident TB Cases & Person Year & Incidence Rate/100 PYO \\
\hline \multirow{4}{*}{ Age Group } & $<=30$ & 31 & 747 & 4.15 \\
\hline & $31-40$ & 10 & 522.08 & 1.9 \\
\hline & $41-50$ & 7 & 166.91 & 4.19 \\
\hline & $>50$ & 5 & 46.56 & 10.7 \\
\hline \multirow{2}{*}{ Sex } & Female & 34 & 881.16 & 3.85 \\
\hline & Male & 19 & 601.39 & 3.15 \\
\hline \multirow{4}{*}{$\mathrm{CD} 4$} & $<=200$ & 29 & 235.06 & 12.3 \\
\hline & 201-350 & 16 & 394.28 & 4.05 \\
\hline & $351-500$ & 4 & 380.95 & 1.05 \\
\hline & $>500$ & 4 & 472.26 & 0.85 \\
\hline \multirow{2}{*}{ BMI } & $>=18.5$ & 18 & 903.38 & 2.0 \\
\hline & $<18.5$ & 35 & 579.17 & 6.04 \\
\hline \multirow{2}{*}{ Weight } & $<=50$ & 36 & 603.07 & 5.96 \\
\hline & $>50$ & 17 & 852.48 & 2.0 \\
\hline \multirow{2}{*}{ WHO Stage } & $\mathrm{I} / \mathrm{II}$ & 36 & 1236.66 & 2.9 \\
\hline & III/IV & 19 & 245.89 & 6.9 \\
\hline \multirow[t]{2}{*}{ Functional status } & Working & 35 & 1271.03 & 2.75 \\
\hline & Ambulatory/ Bedridden & 18 & 211.52 & 8.5 \\
\hline \multirow{2}{*}{ Opportunistic Infection } & Yes & 10 & 295.17 & 3.38 \\
\hline & No & 43 & 1187.38 & 3.6 \\
\hline \multirow{2}{*}{ Cotrimoxazole Treatment } & Yes & 20 & 1064.16 & 1.88 \\
\hline & No & 33 & 418.39 & 7.88 \\
\hline \multirow{2}{*}{ Cohort group } & IPT & 11 & 554.72 & 1.98 \\
\hline & Non-IPT & 42 & 927.83 & 4.52 \\
\hline
\end{tabular}

incidence of TB during the followup period, 3.57 cases per 100 person-years. The TB incidence rate in the IPT group was 1.98 per 100 person-years and 4.52 per 100 person-years for the non-IPT group. Among the TB cases that occurred in the followup period, 34 cases occurred in females giving an incidence in females of 3.85 per 100 person-years and more than half, 31(58.5\%), of the TB cases were diagnosed in those under 30 years old with a crude incidence rate of 4.15 cases per 100 person-years in this age group. During the followup period, the highest TB rate was observed in patients with a CD 4 counts below 200 cells/ul at baseline, accounting for 12.3 cases per 100 person-years (Table 3).

3.3. Predictors of Tuberculosis. After fitting candidate variables into multivariate Cox-regression model, most baseline variables were found to be predictors of TB. PLWHIV (People Living With HIV) at WHO clinical stage III or stage IV had 3.22 times higher risk of acquiring $\mathrm{TB}$ compared to those at WHO clinical stage I or II $(\mathrm{AHR}=3.22,95 \% \mathrm{CI}=1.07-9.7)$. Patients with a baseline CD4 count below 200 cells/ul were fifteen times more likely to develop TB than patients with a CD4 count greater than 500 cells/ul (AHR 15, CI=5.14-43.3). Similarly, PLWHIV with a CD 4 count of between 200 and 350 were 4.6 times more likely to have TB compared to patients with a CD4 count greater than 500 cells/ul (AHR 4.57, CI=1.513.8). Patients who were not treated with cotrimoxazole had
3.47 increased risk of developing TB during the followup compared to treated counterparts $(\mathrm{AHR}=3.47$, 95\%CI 1.88 6.39). Those patients whose body weight was below $50 \mathrm{~kg}$ were at 1.87 increased risk of developing $\mathrm{TB}$ compared to those whose weight was greater than $50 \mathrm{~kg}$ (AHR=1.87, 95\%CI 1.02-3.4). Similarly, patients with a BMI below $18.5 \mathrm{~kg} / \mathrm{m}^{2}$ had a 1.85 greater chance of developing $\mathrm{TB}$ than a patient with a BMI above $18.5 \mathrm{~kg} / \mathrm{m}^{2}$ at baseline $(\mathrm{AHR}=1.85, \mathrm{CI}=1.02-$ 3.55). Patients who were bedridden or whose ambulatory functional status was poor were 2.22 times more likely to develop TB compared to patients who were at working functional status during baseline $(\mathrm{AHR}=2.22, \mathrm{CI}=1.12-4.41)$ (Table 4).

3.4. Impact of IPT. After controlling for other variables, the overall effect of IPT was found to reduce TB incidence by $55 \%(\mathrm{AHR}=0.45, \mathrm{CI}=0.219-0.920)$. In unadjusted bivariate analysis the crude effect of IPT was nearly $60 \%$ (UHR= 0.397, CI=0.203-0.774). Each of the covariate was checked against IPT or IPT was adjusted for each of the covariate independently; none of the covariates altered the effect of IPT by more than $20 \%$. Interaction analysis was also performed to check whether IPT had a differential effect on subgroups of each covariate, but a p-value of the interaction term was statistically insignificant and did not demonstrate any differential effect of IPT on covariates. (Table 5) 
TABLE 4: Predictors of tuberculosis risk, multivariate analysis by Cox proportional hazard model of PLWHA at Nekemte referral hospital and Nekemte Health center followed from September 2009-September 2012.

\begin{tabular}{|c|c|c|c|c|c|}
\hline Variables $(n=600)$ & Category & Unadjusted HR(95\%CI & $\mathrm{P}$-value & Adjusted HR(95\%CI & P-value \\
\hline \multirow{4}{*}{ Age } & $<=30$ & Referent & & Referent & \\
\hline & $31-40$ & $0.47(0.23-0.95)$ & $0.035 *$ & $0.47(0.23-1.05)$ & $0.06^{\#}$ \\
\hline & $41-50$ & $1.05(0.46-2.38)$ & 0.92 & $1.05(0.46-2.38)$ & $0.072^{\#}$ \\
\hline & $>50$ & $2.9(1.13-7.5)$ & $0.027 *$ & $2.9(1.13-7.5)$ & $0.08^{\#}$ \\
\hline \multirow{4}{*}{ CD4 Count } & $>500$ & Referent & & & \\
\hline & $351-500$ & $1.25(0.314-5.16)$ & 0.749 & $1.25(0.89-5.16)$ & $0.68^{\#}$ \\
\hline & $201-350$ & $4.9(1.6-14.6)$ & $0.005 *$ & $4.9(1.6-14.6)$ & 0.007 \\
\hline & $<=200$ & $17.5(6.15-50)$ & $<0.001 *$ & $17.5(6.15-50)$ & $<0.001$ \\
\hline \multirow{2}{*}{ Sex } & Male & Referent & & & \\
\hline & Female & $1.095(0.83-$ & 0.525 & & \\
\hline \multirow{2}{*}{ BMI } & $>=18.5$ & Referent & & referent & \\
\hline & $<18.5$ & $3.13(1.77-5.53)$ & $<0.001 *$ & $3.13(1.77-5.53)$ & 0.042 \\
\hline \multirow{2}{*}{ Weight } & $>50$ & Referent & & Referent & \\
\hline & $<=50$ & $1.72(1.28-2.28)$ & $0.001 *$ & $1.72(1.28-2.28)$ & 0.041 \\
\hline \multirow{2}{*}{ WHO Stage } & I/II & Referent & & Referent & \\
\hline & III/IV & $3.22(0.96-4.56)$ & $0.08 *$ & $3.22(1.96-4.56)$ & 0.037 \\
\hline \multirow{2}{*}{ Functional Status } & Working & Referent & & Referent & \\
\hline & Ambulatory/Bedridden & $3.38(1.9-5.96)$ & $<0.001 *$ & $3.38(1.9-5.96)$ & 0.022 \\
\hline \multirow{2}{*}{ Cotrimoxazole Treatment } & Yes & Referent & & Referent & \\
\hline & No & $4.21(2.42-7.4)$ & $<0.001 *$ & $4.21(2.42-7.4)$ & $<0.001$ \\
\hline \multirow{2}{*}{ Previous TB } & No & Referent & & & \\
\hline & Yes & $1.43(0.56-3.58)$ & 0.45 & & \\
\hline \multirow{2}{*}{ Opportunistic Infection } & No & Referent & & Referent & \\
\hline & Yes & $1.02(0.51-2.02)$ & $0.06 *$ & $1.02(0.51-2.02)$ & $0.061^{\#}$ \\
\hline \multirow{2}{*}{ Cohort Group } & Yes & Referent & & Referent & \\
\hline & No & $2.5(1.3-4.9)$ & $.007 *$ & $2.5(1.3-4.9)$ & 0.03 \\
\hline
\end{tabular}

* All candidate variables for multivariate analysis.

${ }^{\#}$ Lost statistical significance in multivariate analysis.

\section{Discussion}

In this study, the overall incidence of TB was 3.57 per 100 person-years among patients on ART. This is comparable with other studies done in Ethiopia and India, which reported a TB incidence of 3.73 and 2.83 per 100 person-years, respectively, among those on ART $[10,11]$. However, the findings of this study are lower than the findings of the two other studies conducted in Ethiopia which reported 7.0 TB cases per 100 person-years and 8.6 cases per 100 personyears, respectively $[12,13]$. However, these studies were not specifically related to patients on ART which may explain the discrepancy. With prolonged ART, the risk of developing TB is minimized $[14,15]$. In this study, the incidence of TB was higher among patients not on IPT with an incidence of 4.42 per 100 person-years when compared to patients on IPT prophylaxis who had a TB incidence of 1.98 per 100 person-years. This is in agreement with most of the studies conducted in Ethiopia and other countries with higher TB burden $[3,7,8,16-18]$.

This study has also identified several predictive risk factors for TB among HIV infected people enrolled on
ART irrespective of their IPT status. One of the important predictors for $\mathrm{TB}$ occurrence was baseline clinical variables like WHO clinical stage. Even though TB can occur at any HIV clinical stage, it is more common in advanced stages. PLWHIV at WHO clinical stage III or IV were at 3.22 times higher risk of acquiring TB compared to those are at WHO clinical stage I or II. Different authors have demonstrated the positive association between TB incidence and advanced stage of HIV and this has also been found in other studies within Ethiopia [12, 14, 19, 20]. The same result is explained in various studies conducted out of Ethiopia concurring with this study $[8,10,15,17,21]$. Another important finding associated with incident TB was CD4 count below 200 which was found to be a strong predictor of TB. Patients with CD4 count below 200 cells/ul have more risk of developing TB as compared to patients with CD4 count above 500 cells/ul. Similar result is reported by numbers of researches with varying range of risks $[10,12,15]$. Similarly, PLWHIV with a CD 4 count of between 200 and 350 cells/ul were 4.6 times more likely to have TB than a patient with a CD4 count greater than 500 cells/ul. This result was similar to studies done in Ethiopia and which showed there is a two 
TABLE 5: Effect of IPT on reduction of tuberculosis incidence among cohorts of PLWHA at Nekemte referral hospital and Nekemte Health center followed from September 2009-September 2012.

\begin{tabular}{|c|c|c|c|c|c|c|}
\hline IPT adjusted covariates & $\begin{array}{c}\text { Unadjusted } \\
\text { hazard 95\%CI }\end{array}$ & p-value & $\begin{array}{c}\text { Adjusted } \\
\text { Hazard } 95 \% \mathrm{CI}\end{array}$ & $\mathrm{p}$-value & $\begin{array}{c}\text { Significant } \\
\text { reduction in TB } \\
(\%)\end{array}$ & $\begin{array}{l}\mathrm{p} \text {-value for } \\
\text { interaction }\end{array}$ \\
\hline IPT & $\begin{array}{c}0.397(0.203- \\
0.774) \\
\end{array}$ & 0.007 & $\begin{array}{c}0.449(0.219- \\
0.920)^{¥} \\
\end{array}$ & 0.029 & $55^{¥}$ & \\
\hline $\begin{array}{l}\mathrm{IPT} * \\
\text { Cotrimoxazole }\end{array}$ & & & $\begin{array}{c}0.402(0.206- \\
0.784)\end{array}$ & 0.007 & 60 & 0.219 \\
\hline $\begin{array}{l}\mathrm{IPT} * \\
\text { WHO stage } \\
\end{array}$ & & & $\begin{array}{c}0.382(0.196- \\
0.745)\end{array}$ & 0.005 & 62 & 0.48 \\
\hline $\begin{array}{l}\mathrm{IPT} * \\
\mathrm{CD} 4 \text { count }\end{array}$ & & & $\begin{array}{c}0.348(0.176- \\
0.685)\end{array}$ & 0.002 & 65 & 0.64 \\
\hline $\begin{array}{l}\text { IPT } * \\
\text { Functional status }\end{array}$ & & & $\begin{array}{c}0.386(0.198- \\
0.754)\end{array}$ & 0.005 & 61 & 0.08 \\
\hline $\begin{array}{l}\mathrm{IPT} * \\
\mathrm{BMI}\end{array}$ & & & $\begin{array}{c}0.403(0.206- \\
0.788) \\
\end{array}$ & 0.012 & 60 & 0.072 \\
\hline $\begin{array}{l}\text { IPT* } \\
\text { Weight }\end{array}$ & & & $\begin{array}{c}0.426(0.218- \\
0.831)\end{array}$ & 0.008 & 57 & 0.74 \\
\hline $\begin{array}{l}\text { IPT } * \\
\text { Sex }\end{array}$ & & & $\begin{array}{c}0.399(0.204- \\
0.778)\end{array}$ & 0.007 & 40 & 0.85 \\
\hline $\begin{array}{l}\text { IPT } * \\
\text { opportunistic infection }\end{array}$ & & & $\begin{array}{c}0.396(0.203- \\
0.773)\end{array}$ & 0.007 & 60 & 0.61 \\
\hline $\begin{array}{l}\text { IPT* } \\
\text { age }\end{array}$ & & & $\begin{array}{c}0.403(0.206- \\
0.788)\end{array}$ & 0.008 & 60 & 0.065 \\
\hline IPT $*$ previous $\mathrm{TB}$ & & & $\begin{array}{c}0.401(0.205- \\
0.785)\end{array}$ & 0.008 & 60 & 0.588 \\
\hline
\end{tabular}

* IPT adjusted for each covariate.

${ }^{¥}$ Overall effect of IPT adjusted for all covariates.

to three times higher risk in patients with lower CD4 counts [12]. Another important finding of this study was not being treated with cotrimoxazole as an independent predictor of TB. Those patients not treated with cotrimoxazole had 3.47 times greater risk of developing TB than untreated groups (AHR=3.47, 95\%CI 1.88-6.39). This result can confirm that using cotrimoxazole has added benefit in prevention of TB as evidenced in other researches as well [14, 17, 21]. PLWHIV with a baseline body weight below $50 \mathrm{~kg}$ were 1.87 times more likely to have TB than a patient with a body weight above $50 \mathrm{~kg}$. There are also other studies supporting this finding. For example, individuals with a body weight below $50 \mathrm{~kg}$ were more likely to have TB and also prone to failing ART in a study. This shows that patients with higher body weight were less likely to develop TB than those with lower body weights $[4,7,9]$. Additionally, TB cases were more likely to occur in patients with a BMI below 18.5. This finding corresponds with a study conducted in northwest Ethiopia which revealed that patients with a BMI of $<18.5$ at baseline were 1.62 times more likely to get $\mathrm{TB}$ as compared to adults with $\mathrm{BMI} \geq 18.5$ at baseline $[9,10]$. One possible explanation for this is that debilitated patients are more prone to malnutrition and less physical activity and consequently are more vulnerable to many diseases, including TB [9].Another prospective study from Tanzania showed that lower BMI and falling BMI in HIV positive patients was a strong predictor of active TB
[22].This study did not take into account falling BMI and was limited to baseline data due to its retrospective nature. Patients who were bedridden or who had poor ambulatory functional status are more susceptible to develop TB than patients with working functional status during baseline. This is in line with studies from Ethiopia which revealed that the risk of getting TB would be higher if the patient is in a state of poor ambulatory or bedridden with regard to functional status $[12,21]$.

In this study IPT prophylaxis has shown to have a protective effect with a reduction in TB incidence of 55\%; this aligns to other studies conducted in other regions of Ethiopia and other countries with higher TB prevalence $[3,14,16,17]$. One study conducted in Jimma, western Ethiopia, found a $50 \%$ reduction in TB incidence which was solely attributed to IPT used for HIV positive clients [8]. Similarly, another retrospective study in southern Ethiopia within a comparable study setting found the combined effect of IPT and ART, when started simultaneously, reduced TB incidence by $57 \%$ compared to treatment with ART only [19]. Correspondingly, a cross-sectional study conducted in Ethiopia showed IPT prophylaxis combined with cotrimoxazole had a protective benefit against TB with 50\% rate of TB attrition [17]. In contrary to all of these results, a placebo randomized controlled trail (RCT) from Kenya in HIV infected children showed no beneficial effect of IPT [4]. This disparity could be explained 
by the difference in study population when compared to the current study and the retrospective nature of the current study.

\section{Limitations}

The main limitation of this study is the retrospective nature of the cohort. Some important variables like viral load, hemoglobin, adherence status, and ART drug regimen were not included due to problems associated with data inconsistency and incompleteness.

\section{Conclusion and Recommendations}

IPT reduces the risk of TB by $55 \%$ in patients on ART. The study found administration is beneficial even in the absence of TST to diagnose latent TB infection. Further research is required to explore IPT resistance and to look at how many latent cases are going untreated in Ethiopia in the absence of TST.

\section{List of Abbreviations}

AHR: Adjusted hazard ratio

AIDS: Acquired immune deficiency syndrome

ART: Antiretroviral therapy

BMI: $\quad$ Body mass index

CI: $\quad$ Confidence interval

HAART: Highly active antiretroviral therapy

HIV: Human immunodeficiency virus

HR: Hazard ratio

IPT: Isoniaziad preventive therapy

LTBI: Latent tuberculosis infection

PLHIV: People living with human immune deficiency virus

OI: Opportunistic infection

SPSS: $\quad$ Statistical package for social science

TST: Tuberculin skin test

TB: Tuberculosis.

\section{Data Availability}

The data set generated from patients' clinical record is not publicly available to protect patient confidentiality. Unidentifiable data can be obtained from the corresponding author upon reasonable request.

\section{Ethical Approval}

The study was conducted after it was ethically reviewed and approved by the Institutional Review Board (IRB) of the Research Directorate of Wollega University. A letter informing the respective health facility administrators was written by Wollega University and permission was obtained. All information taken from the secondary data source was coded to maintain confidentiality and all personal identifiers were not linked to the data.

\section{Conflicts of Interest}

The authors declare that they have no conflicts of interest regarding the publication of this manuscript.

\section{Authors' Contributions}

Gemechu Tiruneh initiated and designed the study, was involved in collection and analysis of the data, and drafted the manuscript. Emiru Adeba supervised the data extraction process, was involved in data analysis, and reviewed the manuscript. Alemayehu Getahun participated in data analysis and reviewed the manuscript.

\section{Acknowledgments}

We are pleased to thank all health professionals of Nekemte referral hospital and Nekemte health center working in HIV care services. We also want to confer our appreciation to Wollega University College of Health Sciences for offering the chance to undertake the research. Finally, our sincere and special thanks also go to Clare Phillips for editorial commenting of the manuscript.

\section{References}

[1] WHO, "Global Tuberculosis Control Report Geneva," 2015.

[2] WHO, WHO Three I's Meeting Intensified Case Finding (ICF). Isoniazid Preventive Therapy (IPT) and TB Infection Control (IC) for people living with HIV, Report of a Joint World Health Organization HIV/aids and TB Department Meeting, Geneva, SWitzerland, 2008.

[3] J. E. Golub, P. Pronyk, L. Mohapi et al., "Isoniazid preventive therapy, HAART and tuberculosis risk in HIV-infected adults in South Africa: a prospective cohort," AIDS, vol. 23, no. 5, pp. 631-636, 2009.

[4] A. James, A. Lisa, and S. Brett, "Efficacy of isoniazid prophylactic therapy in prevention of tuberculosis in children: a metaanalysis," BMC Infectious Diseases, vol. 14, p. 91, 2014.

[5] S. D. Lawn, R. Wood, K. M. De Cock, K. Kranzer, J. J. Lewis, and G. J. Churchyard, "Antiretrovirals and isoniazid preventive therapy in the prevention of HIV-associated tuberculosis in settings with limited health-care resources," The Lancet Infectious Diseases, vol. 10, no. 7, pp. 489-498, 2010.

[6] G. R. Haileyesus, "Guidelines for intensified tuberculosis casefinding and isoniazid preventive therapy for people living with HIV in resource- constrained settings WHO," 2011.

[7] M. Semu, T. G. Fenta, G. Medhin, and D. Assefa, "Effectiveness of isoniazid preventative therapy in reducing incidence of active tuberculosis among people living with HIV/AIDS in public health facilities of Addis Ababa, Ethiopia: A historical cohort study," BMC Infectious Diseases, vol. 17, no. 1, p. 5, 2017.

[8] L. F. Assebe, H. L. Reda, A. D. Wubeneh, W. T. Lerebo, and S. M. Lambert, "The effect of isoniazid preventive therapy on incidence of tuberculosis among HIV-infected clients under pre-ART care, Jimma, Ethiopia: a retrospective cohort study," BMC Public Health, vol. 15, no. 1, article 346, 2015.

[9] H. Melkamu, B. Seyoum, and Y. Dessie, "Determinants of tuberculosis infection among adult HIV positives attending clinical care in western Ethiopia: a case-control study," AIDS 
Research and Treatment, vol. 2013, Article ID 279876, 7 pages, 2013.

[10] A. Abebe, B. Etana, M. Tsadik, and W. Terefe, "Assessing the effect of highly active anti-retroviral treatment and associated factors on incidence of tuberculosis among adult hiv positive individuals in assela, Ethiopia," Journal of Health \& Medical Informatics, vol. 05, no. 03, Article ID 156, 2014.

[11] S. Rajasekaran, K. Raja, L. Jeyaseelan, S. Vijila, P. Krithiga et al., "Post-HAARTtuberculosis in Adults and adolescents with HIV in India: incidence, clinical andimmunological profile," Indian Journal of Tuberculosis, vol. 56, pp. 69-76, 2009.

[12] K. Addis Alene, A. Nega, and B. Wasie Taye, "Incidence and predictors of tuberculosis among adult people living with human immunodeficiency virus at the University of Gondar Referral Hospital, Northwest Ethiopia," BMC Infectious Diseases, vol. 13, no. 1, p. 292, 2013.

[13] A. Ahmed, D. Mekonnen, and M. Kindie, "Incidence and predictors of tuberculosisamong adult people living with HIV/ AIDS in AfarPublic health facilities, Northeast Ethiopia," AIDS, vol. 1, pp. 3-10, 2015.

[14] C. A. Chang, S. T. Meloni, G. Eisen et al., "Tuberculosis incidence and risk factors among human immunodeficiency virus (HIV)-infected adults receiving antiretroviral therapy in a large HIV program in Nigeria," Open Forum Infectious Diseases, vol. 2, no. 4, Article ID ofv154, 2015.

[15] S. D. Lawn, M. Badri, and R. Wood, “Tuberculosis among HIVinfected patients receiving HAART: Long term incidence and risk factors in a South African cohort," AIDS, vol. 19, no. 18, pp. 2109-2116, 2005.

[16] J. E. Golub, V. Saraceni, S. C. Cavalcante et al., "The impact of antiretroviral therapy and isoniazid preventive therapy on tuberculosis incidence in HIV-infected patients in Rio de Janeiro, Brazil," AIDS, vol. 21, no. 11, pp. 1441-1448, 2007.

[17] A. W. Denegetu and B. L. Dolamo, "Tuberculosis case finding and isoniazid preventive therapy among people living with HIV at public health facilities of Addis Ababa, Ethiopia: A crosssectional facility based study," BMC Public Health, vol. 14, no. 1, p. 52, 2014.

[18] H. T. Ayele, M. S. M. van Mourik, and M. J. M. Bonten, "Effect of isoniazid preventive therapy on tuberculosis or death in persons with HIV: A retrospective cohort study," BMC Infectious Diseases, vol. 15, no. 1, article 334, 2015.

[19] K. D. Yirdaw, D. Jerene, Z. Gashu et al., "Beneficial effect of isoniazid preventive therapy and antiretroviral therapy on the incidence of tuberculosis in people living with HIV in Ethiopia," PLoS ONE, vol. 9, no. 8, Article ID e104557, 2014.

[20] A. Gerardo, R. Pakam, M. Midde, and P. K. Naik, "Incidence and mortality of tuberculosis before and after initiation of antiretroviral therapy: an HIV cohort study in India," Journal of the International Aids Society, vol. 17, no. 1, 2014.

[21] K. T. Kibret, A. W. Yalew, B. G. Belaineh, and M. M. Asres, "Determinant factors associated with occurrence of tuberculosis among adult people living with HIV after antiretroviral treatment initiation in Addis Ababa, Ethiopia: a case control study," PLoS ONE, vol. 8, no. 5, Article ID e64488, 2013.

[22] I. Maro, T. Lahey, T. MacKenzie et al., "Low BMI and falling BMI predict HIV-associated tuberculosis: a prospective study in Tanzania," The International Journal of Tuberculosis and Lung Disease, vol. 14, no. 11, pp. 1447-1453, 2010. 


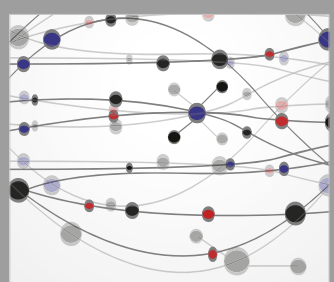

The Scientific World Journal
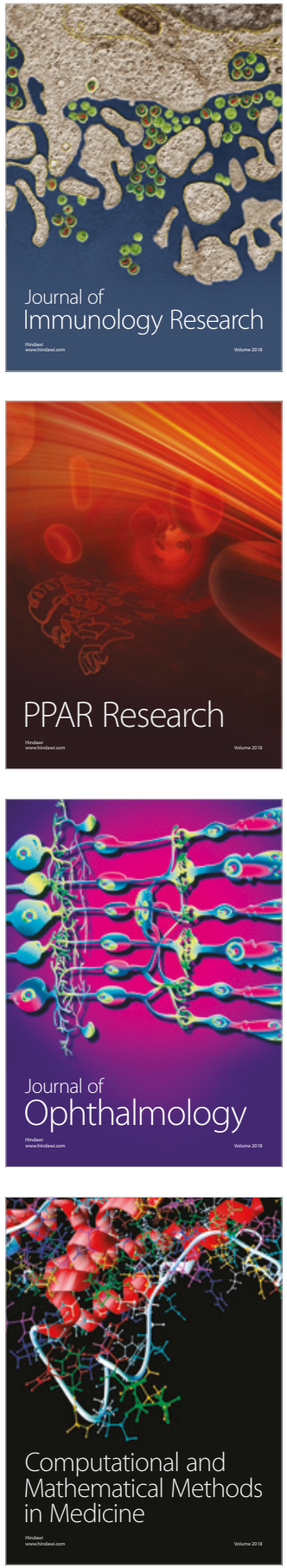

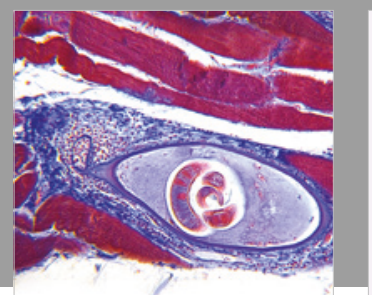

Gastroenterology Research and Practice

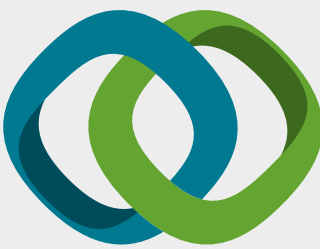

\section{Hindawi}

Submit your manuscripts at

www.hindawi.com
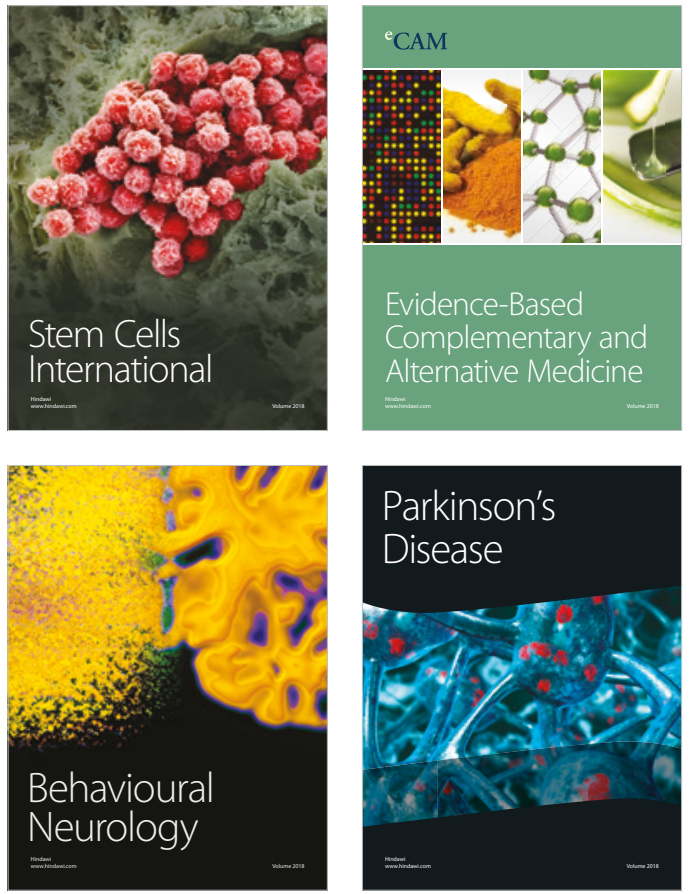

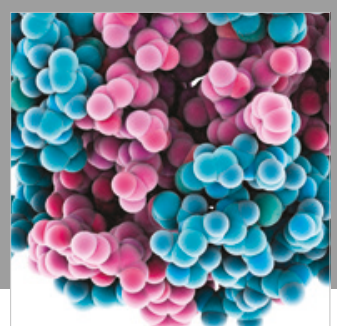

ournal of

Diabetes Research

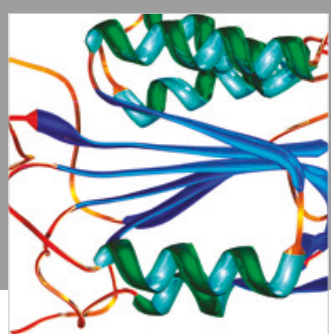

Disease Markers
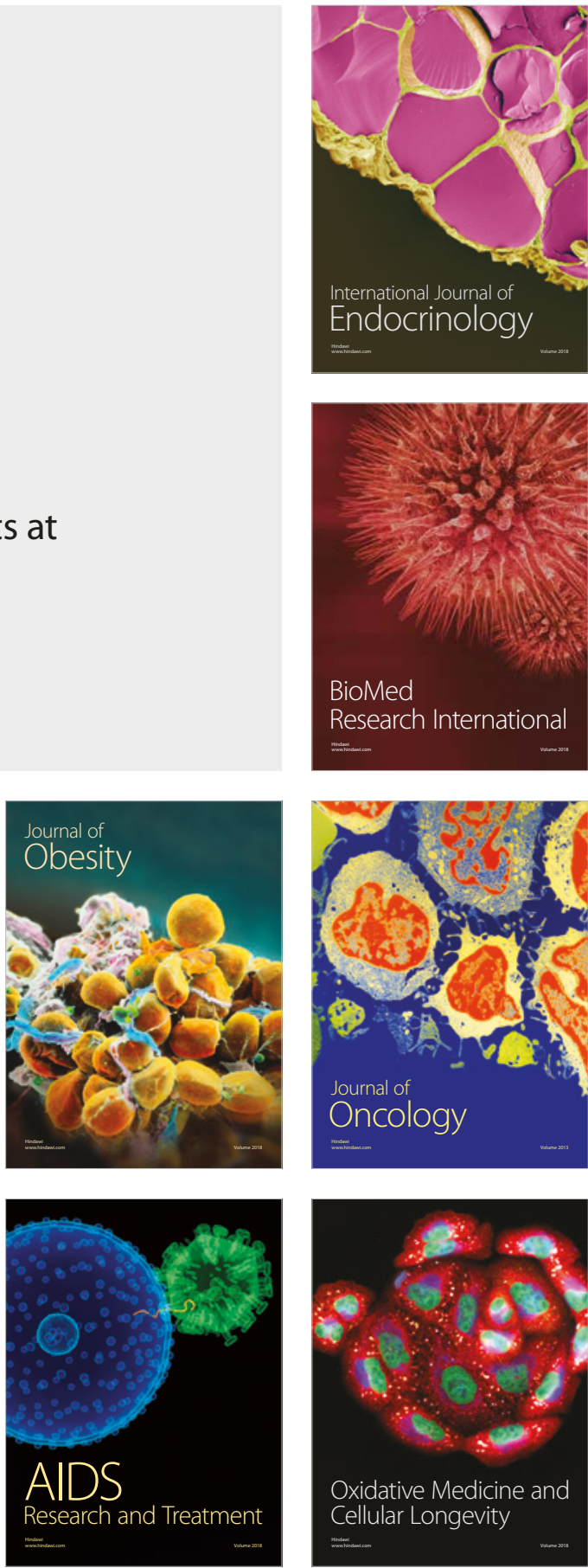